\title{
Limited Feedback-Based Interference Alignment for Interfering Multi-Access Channels
}

\author{
Hui Gao, Member, IEEE, Tiejun Lv, Senior Member, IEEE, Di Fang, Shaoshi Yang, Member, IEEE, and \\ Chau Yuen, Senior Member, IEEE.
}

\begin{abstract}
A limited feedback-based interference alignment (IA) scheme is proposed for the interfering multi-access channel (IMAC). By employing a novel performance-oriented quantization strategy, the proposed scheme is able to achieve the minimum overall residual inter-cell interference (ICI) with the optimized transceivers under limited feedback. Consequently, the scheme outperforms the existing counterparts in terms of system throughput. In addition, the proposed scheme can be implemented with flexible antenna configurations.
\end{abstract}

Index Terms-Interference alignment, interfering multi-access channel, limited feedback.

\section{INTRODUCTION}

Interference alignment (IA) [1], [2] has been considered as a new paradigm of intelligent interference management in wireless networks. The principle of IA is to align the interference signals into a subspace with minimum dimensions at the receiver, and consequently the degrees-of-freedoms (DoFs) of the desired signals can be maximized. Recently, various IA schemes have been developed for the interfering broadcast channel (IBC) and interfering multi-access channel (IMAC) [3]-[6], which are typical models of the cellular networks. It is shown that these IA schemes are capable of improving system throughput in the presence of interference. However, the promise of IA is primarily based on the assumption of global channel state information (CSI) at all transceivers, which requires significant system overhead for CSI.

Aiming at more practical implementations, novel IA schemes with limited feedback have been proposed [7]-[11]. Of particular interests are the IA schemes for the IMAC with limited feedback [7], [8], where the base station (BS) generates the IA-inspired transmit beamforming (TB) vectors with perfect CSI and then feeds back their quantizations to users for uplink transmission. Although [7], [8] assume ideal IA with perfect CSI, the actual inter-cell interference (ICI) cannot be fully canceled at the BS because of the quantization errors.

H. Gao, T. Lv and D. Fang are with the Key Laboratory of Trustworthy Distributed Computing and Service, Ministry of Education, and the School of Information and Communication Engineering, Beijing University of Posts and Telecommunications, Beijing, China 100876 (e-mail: \{huigao, lvtiejun, fangdi\} @bupt.edu.cn). H. Gao is also with Singapore University of Technology and Design, 20 Dover Drive, Singapore 138682.

S. Yang is with the School of Electronics and Computer Science, University of Southampton, SO17 1BJ Southampton, U.K. (e-mail: shaoshi.yang@ @soton.ac.uk).

C. Yuen is with Singapore University of Technology and Design, 20 Dover Drive, Singapore 138682 (email: yuenchau@ @utd.edu.sg).

This work is partially supported by the National Natural Science Foundation of China (NSFC) (Grant No. 61271188) and Singapore University of Technology and Design.
Therefore, the residual ICI is inevitable with limited feedback, which dramatically reduces the achievable system throughput. Moreover, the quantization strategies in [7], [8] are not able to fully exploit the potential of limited feedback. Although the selected codewords are the best approximations of the IAinspired TB vectors within the given codebooks in terms of chordal distance, such codewords are not necessarily the ones that minimize the residual ICI, which directly influences the system performance. Finally, the transceiver designs in [7], [8] are constrained by the feasibility of IA and are only applicable with some antenna configurations.

In this paper, we propose a limited feedback-based IA scheme for the two-cell IMAC. In contrast to [7], [8], the proposed scheme is capable of minimizing the overall residual ICI of each cell with the given codebooks, and it can be implemented with flexible antenna configuration. To be specific, in each cell the optimal receive filter of BS is first derived with an arbitrary set of TB vectors of the users in the neighbor cell, and then the overall residual ICI of each cell is transformed into a single-variable function only regarding the set of TB vectors. Next, each BS jointly quantizes the TB vectors with a compound codebook and a new criterion aiming to directly minimize the overall residual ICI. In this way, the optimized transceivers are obtained under the framework of limited feedback, which effectively approach IA in terms of the minimum overall residual ICI with the given codebooks. Benefiting from the new quantization strategy, the achievable system throughput of our scheme is significantly improved as compared with [7], [8]. Finally, it is worth pointing out that the opportunistic IA in [10], [11] are practical IA schemes with limited feedback, which exploit the multi-user diversity inherited in the cellular networks for throughput gains; the joint design of the proposed scheme with opportunistic user scheduling may be an interesting future direction! 1 .

\section{SYSTEM MODEL}

As illustrated in Fig. 1, a two-cell IMAC is considered, where $\mathrm{BS}_{i}, i \in\{1,2\}$, serves $K$ users $\left\{\mathrm{UE}_{i, k}\right\}_{k=1}^{K}$ in the $i$-th cell. It is assumed that the BS and the user are equipped with $N_{r}\left(K<N_{r}<2 K\right)$ and $N_{t}$ antennas, and each user transmits a single data-stream. The direct and interference channels from $\mathrm{UE}_{i, k}$ to its home $\mathrm{BS}_{i}$ and its neighbor $\mathrm{BS}_{i^{\prime}}$ are denoted as

\footnotetext{
${ }^{1}$ Notation: Bold upper case and lower case letters represent to matrices and vectors, respectively. $(\cdot)^{H}$ denotes the Hermitian transpose and $(\cdot)^{-1}$ represents the inverse of a matrix. $\|\cdot\|$ represent the Frobenius norm of a matrix. $\mathbb{E}[\cdot]$ stands for the expectation. $\rho_{K}(\mathbf{A})$ and $\mathrm{Col}(\mathbf{A})$ denote the sum of the minimum $K$ eigenvalues of $\mathbf{A}$ and the column space of $\mathbf{A}$.
} 


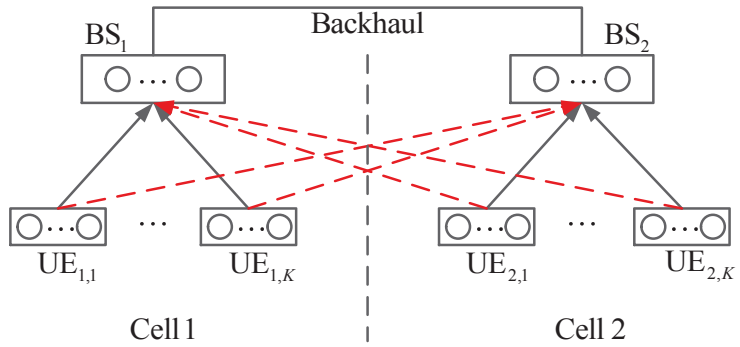

Fig. 1. Two-cell IMAC with limited feeback via backhal. The solid and dashed lines represent the desired and the interfering signals, respectively.

$\mathbf{H}_{i, k} \in \mathbb{C}^{N_{r} \times N_{t}}$ and $\mathbf{G}_{i, k} \in \mathbb{C}^{N_{r} \times N_{t}}$, respectively, $i, i^{\prime} \in$ $\{1,2\}, i^{\prime} \neq i$. The entries of $\mathbf{H}_{i, k}$ and $\mathbf{G}_{i, k}$ are assumed to be independently and identically distributed (i.i.d.) complex Gaussian random variables with zero mean and unit variance, i.e., $\mathcal{C N}(0,1)$. Frequency-division duplexing mode is assumed in this system, and each user sends pilot symbols to both BSs for CSI acquisition. Then $\mathrm{BS}_{i}$ selects the TB vectors from the known codebooks for the users in the neighbor cell by using the estimated CSI of interference channels. The corresponding indices of codewords are exchanged between the two BSs via a backhaul link and then fed back to users to instruct the selection of TB vectors.

During the uplink transmission, all users send information and the received signal at $\mathrm{BS}_{i}$ is given by

$$
\mathbf{y}_{i}=\sum_{k=1}^{K} \mathbf{H}_{i, k} \mathbf{v}_{i, k} s_{i, k}+\sum_{n=1}^{K} \mathbf{G}_{i^{\prime}, n} \mathbf{v}_{i^{\prime}, n} s_{i^{\prime}, n}+\mathbf{n}_{i},
$$

where $\mathbf{v}_{i, k} \in \mathbb{C}^{N_{t} \times 1}$ and $s_{i, k} \in \mathbb{C}$ respectively denote the normalized $\mathrm{TB}$ vector chosen from $\mathrm{UE}_{i, k}$ 's codebook and the transmitted symbol of $\mathrm{UE}_{i, k}$ with an average power of $P$; $\mathbf{n}_{i} \in \mathbb{C}^{N_{r} \times 1}$ is the noise vector with i.i.d. elements following $\mathcal{C N}\left(0, N_{0}\right)$. The receiver at $\mathrm{BS}_{i}$ is a cascaded filter $\mathbf{r}_{i, k}^{H} \mathbf{U}_{i}^{H}$, where $\mathbf{U}_{i} \in \mathbb{C}^{N_{r} \times K}$ and $\mathbf{r}_{i, k} \in \mathbb{C}^{K \times 1}$ are designed to cancel the ICI and the intra-cell interference for $\mathrm{UE}_{i, k}$. The column vectors of $\mathbf{U}_{i}$ are normalized and orthogonal to each other. Let us define the effective channel matrix from all the users $\left\{\mathrm{UE}_{i, k}\right\}_{k=1}^{K}$ to $\mathrm{BS}_{i}$ as $\mathbf{H}_{i, e}=\mathbf{U}_{i}^{H}\left[\mathbf{H}_{i, 1} \mathbf{v}_{i, 1}, \ldots, \mathbf{H}_{i, K} \mathbf{v}_{i, K}\right] \in$ $\mathbb{C}^{K \times K}, \mathbf{r}_{i, k}^{H}$ is then given by the $k$-th normalized row vector of $\mathbf{H}_{i, e}^{-1}$. Based on (1) and the cascaded filter $\mathbf{r}_{i, k}^{H} \mathbf{U}_{i}^{H}$, the throughput of $\mathrm{UE}_{i, k}$ is given by

$$
R_{i, k}=\log \left(1+\frac{\operatorname{SNR}\left|\mathbf{r}_{i, k}^{H} \mathbf{U}_{i}^{H} \mathbf{H}_{i, k} \mathbf{v}_{i, k}\right|^{2}}{1+\operatorname{SNR} \sum_{n=1}^{K}\left|\mathbf{r}_{i, k}^{H} \mathbf{U}_{i}^{H} \mathbf{G}_{i^{\prime}, n} \mathbf{v}_{i^{\prime}, n}\right|^{2}}\right),
$$

where $\mathrm{SNR}=\frac{P}{N_{0}}$ denotes the transmit signal-to-noise ratio (SNR) and $\sum_{n=1}^{K}\left|\mathbf{r}_{i, k}^{H} \mathbf{U}_{i}^{H} \mathbf{G}_{i^{\prime}, n} \mathbf{v}_{i^{\prime}, n}\right|^{2}$ is considered as the final residual $\mathrm{ICl}^{2}$. It is observed from (2) that the residual ICI reduces the system throughput.

\footnotetext{
${ }^{2}$ It is noted that the actual throughput of IA with limited feedback can almost always be formulated as (2), regardless of the specific IA-inspired TB vectors [7], [8] before quantization. In addition, we also keep the notation of residual ICI to highlight the transmission with limited feedback, even though we bypass the IA-inspired TB vectors employed in [7], [8].
}

\section{Limited FEEDBACK-BASEd IA SCHEME}

\section{A. Transceiver Design with Limited Feedback}

It is noted that $\mathrm{Col}\left(\mathbf{U}_{i}\right)$ is a $K$-dimension signal subspace where the desired signals of the $K$ users $\left\{\mathrm{UE}_{i, k}\right\}_{k=1}^{K}$ are further differentiated by $\left\{\mathbf{r}_{i, k}\right\}_{k=1}^{K}$. Because of the imperfect IA with limited feedback, $\mathrm{Col}\left(\mathbf{U}_{i}\right)$ is inevitably contaminated by the residual ICI. For analytical tractability, we introduce the overall residual ICI within $\mathrm{Col}\left(\mathbf{U}_{i}\right)$ at $\mathrm{BS}_{i}$ as

$$
I_{i}=\sum_{n=1}^{K}\left\|\mathbf{U}_{i}^{H} \mathbf{G}_{i^{\prime}, n} \mathbf{v}_{i^{\prime}, n}\right\|^{2} \geq \sum_{n=1}^{K}\left|\mathbf{r}_{i, k}^{H} \mathbf{U}_{i}^{H} \mathbf{G}_{i^{\prime}, n} \mathbf{v}_{i^{\prime}, n}\right|^{2},
$$

which is shown to be an upper bound of the final residual ICI power after applying $\mathbf{r}_{i, k}^{H} \sqrt[3]{3}$. It is observed that $I_{i}$ contains the receive filter $\mathbf{U}_{i}$ and the set of the $K$ TB vectors, denoted as $\mathbf{V}_{i^{\prime}}:=\left\{\mathbf{v}_{i^{\prime}, n}\right\}_{n=1}^{K}$. Therefore, both $\mathbf{U}_{i}$ and $\mathbf{V}_{i^{\prime}}$ can be optimized to minimize $I_{i}$ for an improved system performance under limited feedback.

Unlike the quantization strategies in [7], [8], which independently choose $\mathbf{v}_{i^{\prime}, n}$ from $\mathrm{UE}_{i, k}$ 's individual codebook to approximate its IA-inspired TB vector designed with perfect CSI, we aims to jointly choose $\mathbf{V}_{i^{\prime}}$ from an new compound codebook to directly minimize the overall residual ICI $I_{i}$. It is noted that although [7], [8] may find the best quantization for each IA-inspired TB vector, the selected codewords are not able to directly minimize the residual ICI by jointly optimizing $\mathbf{U}_{i}$ and $\mathbf{V}_{i^{\prime}}$. Noting the potential to further improve system performance with optimized $\mathbf{U}_{i}$ and $\mathbf{V}_{i^{\prime}}$, we base our IA transceiver design directly on the overall residual ICI without the reference or constraint of the IA-inspired TB as [7], [8], and our limited feedback-based IA can fully utilize the given codebooks to achieve the minimal overall residual ICI $I_{i}$.

To start with, we aims to transfer the objective $I_{i}$ into a single variable function only regarding $\mathbf{V}_{i^{\prime}}$. This is achieved by first deriving the structure of the optimal $\mathbf{U}_{i}$ with arbitrary $\mathbf{V}_{i^{\prime}}$. Let $\mathbf{U}_{i}=\left[\mathbf{u}_{i, 1}, \ldots, \mathbf{u}_{i, K}\right]$, according to the rotation invariance property of Frobenius norm [12], we have

$$
I_{i}\left(\mathbf{U}_{i}, \mathbf{V}_{i^{\prime}}\right)=\sum_{n=1}^{K} \mathbf{u}_{i, n}^{H} \mathbf{A}_{i^{\prime}}\left(\mathbf{V}_{i^{\prime}}\right) \mathbf{u}_{i, n}
$$

where $\mathbf{A}_{i^{\prime}}\left(\mathbf{V}_{i^{\prime}}\right):=\tilde{\mathbf{G}}_{i^{\prime}}\left(\mathbf{V}_{i^{\prime}}\right) \tilde{\mathbf{G}}_{i^{\prime}}^{H}\left(\mathbf{V}_{i^{\prime}}\right)$ is introduced for simplicity and $\tilde{\mathbf{G}}_{i^{\prime}}\left(\mathbf{V}_{i^{\prime}}\right)=\left[\mathbf{G}_{i^{\prime}, 1} \mathbf{v}_{i^{\prime}, 1}, \ldots, \mathbf{G}_{i^{\prime}, K} \mathbf{v}_{i^{\prime}, K}\right] \in \mathbb{C}^{N_{r} \times K}$ is the compound interfering channel at $\mathrm{BS}_{i}$ before further processing. Let us define the ascendingly ordered eigenvalues of $\mathbf{A}_{i^{\prime}}\left(\mathbf{V}_{i^{\prime}}\right)$ as $\lambda_{1}\left(\mathbf{V}_{i^{\prime}}\right), \ldots, \lambda_{N_{r}}\left(\mathbf{V}_{i^{\prime}}\right)$ and the corresponding normalized eigenvectors as $\mathbf{w}_{1}\left(\mathbf{V}_{i^{\prime}}\right), \ldots, \mathbf{w}_{N_{r}}\left(\mathbf{V}_{i^{\prime}}\right)$. For any given $\mathbf{V}_{i^{\prime}}, I_{i}\left(\mathbf{U}_{i}, \mathbf{V}_{i^{\prime}}\right)$ is minimized when $\mathbf{u}_{i, n}\left(\mathbf{V}_{i^{\prime}}\right)=$ $\mathbf{w}_{n}\left(\mathbf{V}_{i^{\prime}}\right), n=1, \ldots, K$ [12], then the optimal $\mathbf{U}_{i}$ can be defined as a function of $\mathbf{V}_{i^{\prime}}$ as

$$
\mathbf{U}_{i}^{*}\left(\mathbf{V}_{i^{\prime}}\right)=\left[\mathbf{w}_{1}\left(\mathbf{V}_{i^{\prime}}\right), \ldots, \mathbf{w}_{K}\left(\mathbf{V}_{i^{\prime}}\right)\right] .
$$

Applying $\mathbf{U}_{i}^{*}\left(\mathbf{V}_{i^{\prime}}\right), I_{i}$ can be reformulated as

$$
I_{i}\left(\mathbf{U}_{i}^{*}\left(\mathbf{V}_{i^{\prime}}\right), \mathbf{V}_{i^{\prime}}\right)=I_{i}\left(\mathbf{V}_{i^{\prime}}\right)=\rho_{K}\left(\mathbf{A}_{i^{\prime}}\left(\mathbf{V}_{i^{\prime}}\right)\right) .
$$

${ }^{3}$ The inequality in (3) can be easily proved by using the property $\|\mathbf{a}\|\|\mathbf{b}\| \geq$ $\left|\mathbf{a}^{H} \mathbf{b}\right|$ with $\|\mathbf{a}\|=1$. 
Comparing (4) and (6), it is observed that the original objective function $I_{i}\left(\mathbf{U}_{i}, \mathbf{V}_{i^{\prime}}\right)$ has been transferred to $I_{i}\left(\mathbf{V}_{i^{\prime}}\right)$ with $\mathbf{U}_{i}^{*}\left(\mathbf{V}_{i^{\prime}}\right)$, which is a single-variable function regarding $\mathbf{V}_{i^{\prime}}$ or the quantized TB vectors $\left\{\mathbf{v}_{i^{\prime}, n}\right\}_{n=1}^{K}$.

Focusing directly on the objective function $I_{i}\left(\mathbf{V}_{i^{\prime}}\right)$, we propose a joint quantization strategy to choose the optimal $\mathbf{V}_{i^{\prime}}$ from a compound codebook. As a very brief review and preliminary, it is noted that in [7], [8] $\mathbf{v}_{i^{\prime}, n}$ is individually selected (but not optimized) by $\mathrm{BS}_{i}$ from the randomly generated codebook $\mathcal{C}_{i^{\prime}, n}=\left\{\mathbf{c}_{i^{\prime}, n, 1}, \mathbf{c}_{i^{\prime}, n, 2}, \ldots, \mathbf{c}_{i^{\prime}, n, 2^{B}}\right\}$ of $\mathrm{UE}_{i, k}$ with the minimum chordal distance criterion, where $\mathbf{c}_{i^{\prime}, n, m} \in \mathbb{C}^{N_{t} \times 1}$ is the normalized codeword with the index $m \in\left\{1,2, \ldots, 2^{B}\right\}$ and $B$ denotes the number of feedback bits per user. Unlike [7], [8], we enlarge the size of the individual codebook of each user from $2^{B}$ to $2^{K B}$, and jointly select the TB vectors $\left\{\mathbf{v}_{i^{\prime}, n}\right\}_{n=1}^{K}$ to directly minimize $I_{i}$ with a new strategy. More specifically, we group every $m$-th codeword from each $\mathcal{C}_{i^{\prime}, n}, n=1,2, \ldots, K$, into a compound codeword as $\mathbf{C}_{i^{\prime},(m)}=\left\{\mathbf{c}_{i^{\prime}, 1, m}, \ldots, \mathbf{c}_{i^{\prime}, K, m}\right\}$ [13], then we collect the $2^{K B}$ compound codewords into a new codebook $\mathcal{C}_{i^{\prime}}=\left\{\mathbf{C}_{i^{\prime},(1)}, \mathbf{C}_{i^{\prime},(2)}, \ldots, \mathbf{C}_{i^{\prime},\left(2^{K B}\right)}\right\}$ for the joint quantization

$$
\mathbf{V}_{i^{\prime}}^{*}=\left\{\mathbf{v}_{i^{\prime}, 1}^{*}, \ldots, \mathbf{v}_{i^{\prime}, K}^{*}\right\}=\arg \min _{\mathbf{V}_{i^{\prime}} \in \mathcal{C}_{i^{\prime}}} \rho_{K}\left(\mathbf{A}_{i^{\prime}}\left(\mathbf{V}_{i^{\prime}}\right)\right) \text {. }
$$

It is worth pointing out that after the joint quantization, each BS only needs to exchange and fed back one common index $m_{i^{\prime}}^{*}$ corresponding with $\mathbf{V}_{i^{\prime}}^{*}$ to all the served $K$ users at the cost of $K B$ bits, which means the total feedback per cell is still the same as [7], [8]. Upon receiving $m_{i^{\prime}}^{*}, \mathrm{UE}_{i^{\prime}, k}$ uses $\mathbf{v}_{i^{\prime}, k}^{*}=\mathbf{c}_{i^{\prime}, k, m_{i^{\prime}}^{*}}$ as the TB vector and $\mathrm{BS}_{i}$ uses $\mathbf{U}_{i}^{*}\left(\mathbf{V}_{i^{\prime}}^{*}\right)$ to establish its cascaded receive filter.

Finally, the complexity of the proposed scheme is briefly discussed. It is observed (7) mainly includes the construction of $\mathbf{A}_{i^{\prime}}\left(\mathbf{V}_{i^{\prime}}\right)$ and its singular value decomposition, which cost $2 K N_{r}\left(3 N_{t}-1\right)$ floating point operations (flops) and $O\left(N_{r} K^{2}\right)$ flops [14], respectively. Since the searching space has a size of $2^{K B}$, it is easy to estimate the overall complexity of the proposed scheme as $O\left(2^{K B} N_{r} K\left(3 N_{t}+K-1\right)\right)$. Although our scheme is more complicated than [7], [8], such computation overhead is still affordable at the BS. Moreover, our scheme shows significant throughput gain as compared to [7], [8], which will be validated in the following sections.

Remark 1: Intuitively, [7], [8] can be considered as the traditional IA schemes with limited feedback. More specifically, the IA-inspired TB vectors are designed before quantization and the achievability of IA mainly relies on the qualities of quantization. However, such quantization aims to approach the IA-inspired TB vectors but not necessarily to the best achievable throughput performance with the given codebooks. In contrast to [7], [8], we bypass the IA-inspired transceivers before quantization, and we straightforwardly approach IA in terms of the minimal residual ICI by finding the most appropriate codewords within the given codebook. In this sense, our scheme is specifically designed for limited feedback, and it is therefore a limited feedback-based IA scheme. In addition, our scheme is not constrained by the feasibility condition for the IA-inspired transceivers, and can be implemented with flexible antenna configurations.

\section{B. Performance Analysis}

In this section, we aims to initially analyze the performance the proposed scheme. For the comparison purpose, we use [7] as a reference and set $N_{t}=N_{r}$ accordingly. Then the throughput of $\mathrm{UE}_{i, k}$ with perfect feedback is given as

$$
R_{i, k}^{P F B}=\log _{2}\left(1+\operatorname{SNR}\left|\tilde{\mathbf{r}}_{i, k}^{H} \tilde{\mathbf{U}}_{i}^{H} \mathbf{H}_{i, k} \tilde{\mathbf{v}}_{i, k}\right|^{2}\right),
$$

where $\tilde{\mathbf{r}}_{i, k}, \tilde{\mathbf{U}}_{i}$ and $\tilde{\mathbf{v}}_{i, k}$ are obtained by using the IAinspired transceiver design of [7] without quantization. Since $R_{i, k}^{P F B}$ assumes the ICI-free scenario, it can be equivalently considered as the ideal throughput of the proposed scheme for this comparison. Based on $R_{i, k}^{P F B}$ and $R_{i, k}$ in (6), the throughput loss of $\mathrm{UE}_{i, k}$ with our scheme is defined as

$$
\Delta R_{i, k}=R_{i, k}^{P F B}-R_{i, k} .
$$

The following theorem gives a upper bound of $\mathbb{E}\left[\Delta R_{i, k}\right]$ to better understand the gains of the proposed scheme.

Theorem 1: When $N_{t}=N_{r}$, the upper bound of the average throughput loss of $\mathrm{UE}_{i, k}$ with the proposed scheme is

$$
\mathbb{E}\left[\Delta R_{i, k}\right] \leq \log _{2}\left(1+\operatorname{SNR} \mathbb{E}\left[\min _{m \in\left\{1, \ldots, 2^{K B}\right\}} \rho_{K}\left(\mathbf{A}_{(m)}\right)\right]\right),
$$

where $\left\{\mathbf{A}_{(m)}, m \in\left\{1, \ldots, 2^{K B}\right\}\right\}$ are some i.i.d. complex central Wishart matrices which follow $\mathcal{C W}_{N_{r}}\left(K, \mathbf{I}_{N_{r}}\right)$.

Proof: We first establish the following inequalities,

$$
\begin{aligned}
\mathbb{E}\left[\Delta R_{i, k}\right] & \leq \log _{2}\left(1+\mathbb{E}\left[\operatorname{SNR} \sum_{n=1}^{K}\left|\mathbf{r}_{i, k}^{H} \mathbf{U}_{i}^{H} \mathbf{G}_{i^{\prime}, n} \mathbf{v}_{i^{\prime}, n}\right|^{2}\right]\right) \\
& \leq \log _{2}\left(1+\frac{\mathbb{E}\left[I_{i}\right]}{N_{0}}\right) \\
& =\log _{2}\left(1+\operatorname{SNR} \mathbb{E}\left[\min _{m \in\left\{1, \ldots, 2^{K B}\right\}} \rho_{K}\left(\mathbf{A}_{(m)}\right)\right]\right)
\end{aligned}
$$

where the first inequality follows (6) of [7], the second inequality follows the inequality in (3), and last equation follows (6) and (7) by treating $\mathbf{A}_{i^{\prime}}\left(\mathbf{C}_{i^{\prime},(m)}\right)$ as $\mathbf{A}_{(m)}$. Next, we continue to show the distribution of $\mathbf{A}_{(m)}$. Since $\mathbf{c}_{i^{\prime}, n, m}$ is a normalized vector and the entries of $\mathbf{G}_{i^{\prime}, n}$ are i.i.d. $\mathcal{C N}(0,1)$, the entries of $\mathbf{G}_{i^{\prime}, n} \mathbf{c}_{i^{\prime}, n, m}$ are i.i.d. $\mathcal{C N}(0,1)$. Then it is easy to show that $\mathbf{A}_{(m)}$ is a complex central Wishart matrix which follows $\mathcal{C W}_{N_{r}}\left(K, \mathbf{I}_{N_{r}}\right)$ [15]. Since the codewords $\mathcal{C}_{i^{\prime}}$ are i.i.d., it is easy to show that $\left\{\tilde{\mathbf{G}}_{i^{\prime}}\left(\mathbf{C}_{i^{\prime},(m)}\right), \mathbf{C}_{i^{\prime},(m)} \in \mathcal{C}_{i^{\prime}}\right\}$ are also i.i.d., and then the proof is finished.

Based on Theorem 1 and (9), a lower bound of the average throughput with the proposed scheme can be obtained as

$$
\begin{aligned}
\mathbb{E}\left[R_{i, k}\right] \geq & \mathbb{E}\left[R_{i, k}^{P F B}\right] \\
& -\log _{2}\left(1+\mathrm{SNR} \cdot \mathbb{E}\left[\min _{m \in\left\{1, \ldots, 2^{K B}\right\}} \rho_{K}\left(\mathbf{A}_{(m)}\right)\right]\right)
\end{aligned}
$$

It is noted that the joint density of the eigenvalues of complex central Wishart matrix is given by (19) of [15]. However, the distribution of $\min _{m \in\left\{1, \ldots, 2^{K B}\right\}} \rho_{K}\left(\mathbf{A}_{(m)}\right)$ is still very complicated. Therefore, we resort to the numerical method to evaluate the lower bound in (12) for the comparison with [7], which can be simulated faster than $\mathbb{E}\left[R_{i, k}\right]$. 


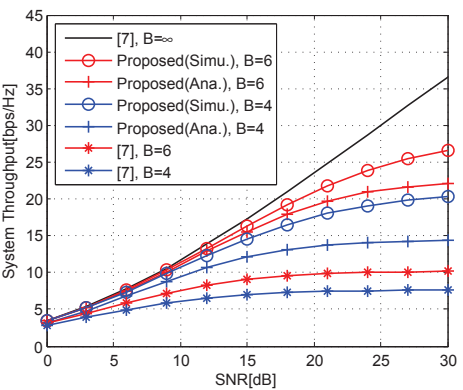

Fig. 2. System throughput comparison with [7], $N_{t}=N_{r}=3, K=2$.

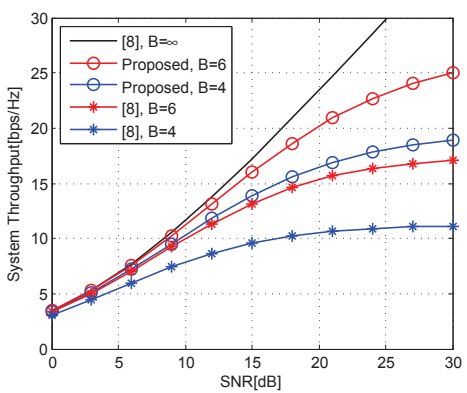

Fig. 3. System throughput comparison with [8], $N_{t}=2, N_{r}=3, K=2$.

Remark 2: For the comparison purpose with [7], the above analysis only focus on the configuration $N_{t}=N_{r}$. Although our scheme is applicable with arbitrary $N_{t}$, it is often assumed that the users have less antennas than the BS. When $N_{t}<N_{r}$, it is noted the elements in $\left\{\mathbf{A}_{(m)}, m \in\left\{1, \ldots, 2^{K B}\right\}\right\}$ are not independent, and the statistical analysis is more involved and is deferred as future work.

\section{Simulation Results}

We first validate the theoretical results related to Theorem 1 and compare our scheme with [7]. The system configuration are set as $N_{t}=N_{r}=3, K=2$, and the number of feedback bits or $B$ is specified with the simulation curves. In Fig. 2, the inequality (12) are demonstrated, where the simulation results are obtained by performing the proposed scheme, and the analytical results are calculated according to the lower bound in (12). It is shown that the lower bound of the proposed scheme has already exceeded the achievable system throughput of [7]. Therefore, the throughput gains of the proposed scheme are proved.

Then we compare the proposed scheme with [8] under a variety of system configurations. As shown in Fig. 3, given the same number of feedback bits $B=4,6$, the proposed scheme achieves significant gain over [8] with the system configuration $N_{t}=2, N_{r}=3, K=2$. Moreover, it is noted that due to the IA-feasibility constraint, [8] is not applicable with the system configuration $N_{r}=4, K=3$ and $N_{t} \leq \frac{3}{4} K$, while our scheme is still applicable with arbitrary $N_{t}$, as shown in Fig. 4. It is also observed that the throughput performance of our scheme increase with $N_{t}$ when $N_{r}, K$ and $B$ are fixed, which shows the benefit of extra transmit antennas at user.

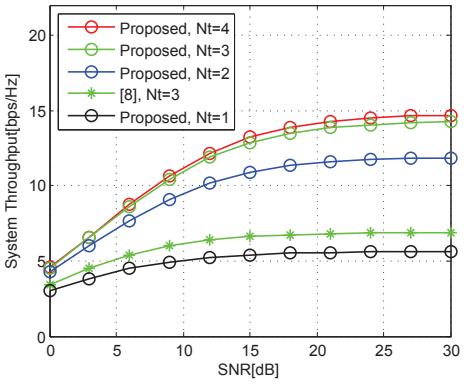

Fig. 4. System throughput comparison with [8], $N_{r}=4, K=3, B=4$.

\section{CONCLUSION}

In this paper, a limited feedback-based IA scheme has been proposed for the IMAC. The optimized transceivers have been designed with the performance-oriented quantization strategy to minimize the residual ICI. As a beneficial result, the proposed scheme achieves a significant gain of system throughput and can be implemented with flexible antenna configurations.

\section{REFERENCES}

[1] V. R. Cadambe and S. A. Jafar, "Interference alignment and degrees of freedom of the K-user interference channel," IEEE Trans. Inf. Theory, vol. 54, pp. 3425-3441, Aug. 2008.

[2] M. Maddah-Ali, A. Motahari, and A. Khandani, "Communication over MIMO X channels: Interference alignment, decomposition, and performance analysis," IEEE Trans. Inf. Theory, vol. 54, pp. 3457-3470, Aug. 2008.

[3] C. Suh and D. Tse, "Interference alignment for cellular networks," in Proc. Allerton Conf. on Comm., Cont., and Comp., Berkeley, CA, Sept. 2008, pp. 1037-1044.

[4] C. Suh, M. Ho, and D. Tse, "Downlink interference alignment," IEEE Trans. Commun., vol. 59, pp. 2616-2626, Sept. 2011.

[5] W. Shin, N. Lee, J.-B. Lim, C. Shin, and K. Jang, "On the design of interference alignment scheme for two-cell MIMO interfering broadcast channels," IEEE Trans. Wireless Commun., vol. 10, pp. 437-442, Feb. 2011.

[6] H.-H. Lee, M.-J. Kim, and Y.-C. Ko, "Transceiver design based on interference alignment for MIMO interfering broadcast channels," in Proc. IEEE GLOBECOM, Anaheim, CA, Dec. 2012, pp. 5266-5271.

[7] S. Cho, K. Huang, D. Kim, and H. Seo, "Interference alignment for uplink cellular systems with limited feedback," IEEE Commun. Lett., vol. 16, pp. 960-963, July 2012.

[8] N. Lee, W. Shin, R. W. Heath Jr., and B. Clerckx, "Interference alignment with limited feedback for two-cell interfering MIMO-MAC," in Proc. IEEE ISWCS, Aug. 2012, pp. 566-570.

[9] X. Chen and C. Yuen, "Performance analysis and optimization for interference alignment over MIMO interference channels with limited feedback," IEEE Trans. Signal Process., vol. 99, pp. 1-10, Feb. 2014.

[10] J. Leithon, C. Yuen, H. A. Suraweera, and H. Gao, "A new opportunistic interference alignment scheme and performance comparison of MIMO interference alignment with limited feedback," in Proc. IEEE GLOBECOM Workshop on Multicell Cooperation, Anaheim, CA, Dec. 2012, pp. $1123-1127$.

[11] H. Gao, J. Leithon, C. Yuen, and H. A. Suraweera, "New uplink opportunistic interference alignment: An active alignment approach," in Proc. IEEE WCNC, Shanghai, China, Apr. 2013, pp. 1123-1127.

[12] R. A. Horn and C. R. Johnson, Matrix Analysis. Cambridge University Press, 1985.

[13] D. Fang, S. Su, T. Lv, H. Gao, and W. Long, "Joint quantization strategy for uplink cooperative cellular interference alignment with limited feedback," in Proc. IEEE MILCOM, Orlando, FL, Oct. 2012, pp. $1-5$.

[14] G. H. Golub and C. F. V. Loan, Matrix Computations. Johns Hopkins University Press, 1996.

[15] T. Ratnarajah, R. Vaillancourt, and M. Alvo, "Eigenvalues and condition numbers of complex random matrices," SIAM Journal on Matrix Analysis and Applications, vol. 26, no. 2, pp. 441-456, 2004. 